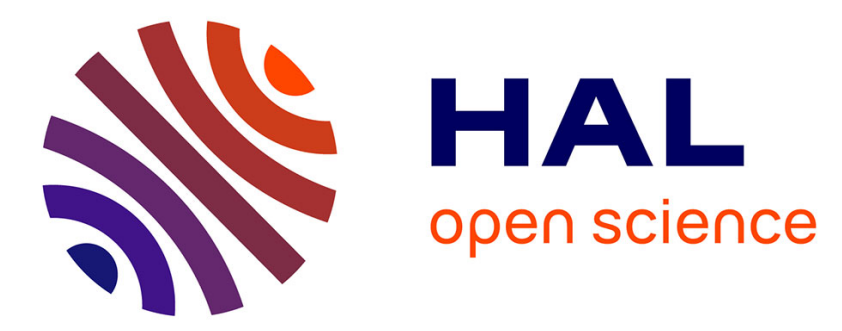

\title{
A strategy for simulating Electron Energy-Loss Near-Edge Structures of nanoparticles: application to size effects in GdO
}

\author{
T. Épicier, J.-C. Le Bossé, P. Perriat, S. Roux, O. Tillement
}

\section{To cite this version:}

T. Épicier, J.-C. Le Bossé, P. Perriat, S. Roux, O. Tillement. A strategy for simulating Electron Energy-Loss Near-Edge Structures of nanoparticles: application to size effects in GdO. European Physical Journal: Applied Physics, 2011, 54 (3), pp.33511. 10.1051/epjap/2011100440 . hal-00706165

\section{HAL Id: hal-00706165 \\ https://hal.science/hal-00706165}

Submitted on 9 Jun 2012

HAL is a multi-disciplinary open access archive for the deposit and dissemination of scientific research documents, whether they are published or not. The documents may come from teaching and research institutions in France or abroad, or from public or private research centers.
L'archive ouverte pluridisciplinaire HAL, est destinée au dépôt et à la diffusion de documents scientifiques de niveau recherche, publiés ou non, émanant des établissements d'enseignement et de recherche français ou étrangers, des laboratoires publics ou privés. 


\title{
A strategy for simulating Electron Energy-Loss Near-Edge Structures of nanoparticles: application to size effects in $\mathbf{G d}_{2} \mathbf{O}_{3}$
}

Thierry Épicier ${ }^{1}$, Jean-Claude Le Bossé ${ }^{1}$, Pascal Perriat ${ }^{1}$, Stéphane Roux $^{2}$ and Olivier Tillement ${ }^{2}$

INSA-Lyon, MATEIS CNRS UMR5510, bat. Blaise Pascal, F-69621 Villeurbanne Cedex

Université de Lyon, Université Lyon 1, LPCML CNRS UMR5620, F-69622 Villeurbanne Cedex

Received: 01/11/2010 / Revised version: date

\begin{abstract}
Nanoparticles (NPs) have attracted a considerable interest in the last decades, owing to their remarkable physical and chemical properties. The most important characteristic of NPs is the size effect, that is their properties differ from that of the corresponding bulk material. We will focus here on Electron Energy-Loss Spectroscopy (EELS) investigations of $\mathrm{Gd}_{2} \mathrm{O}_{3}$ NPs of different and controlled sizes. EELS spectra near the $\mathrm{O} \mathrm{K}$ edge of $\mathrm{Gd}_{2} \mathrm{O}_{3}$ were recorded and compared with feff8.2 ELNES simulations. The calculation of the EELS response from small particles by the feff code raises some particular problems which have been carefully examined and partially solved. The simulations are in fair agreement with experiment and reveal the existence of size effects.
\end{abstract}

\section{Introduction}

\subsection{Position of the problem}

Nanoparticles (NPs) constitute today a class of materials of considerable interest for a very wide range of applications in many fields. Their unique physical and chemical properties, including structural, electric, magnetic, thermodynamic or catalytic aspects, mostly arise from the fact that the ratio between surface and bulk atoms is obviously Send offprint requests to: thierry.epicier0insa-lyon.fr much higher than in bulk materials. This size effect has been investigated in a large number of situations (see, for example, works related to structural properties [1-3] or to recent applications such as in catalysis [4] or toxicol$\operatorname{ogy}[5])$.

The aim of the present work is to illustrate such a size effect by means of Electron Energy Loss Spectroscopy (EELS). Owing to spatial and energetic resolutions of modern (Scanning) Transmission Electron Microscopes, EELS is a very powerful method to probe NPs at a very local 
scale, as was for example recently demonstrated in the study of excitation of various plasmon modes in metallic particles $[6,7]$. However probing single NPs at the atomic level remains very delicate from an experimental point of view (see below); a strategy has thus to be established in order to calculate the EELS response of a set of NPs randomly oriented on a supporting film or randomly embedded in a matrix. Indeed considering NPs or any nanosized objects such as nanotubes or nanowires raises different problems regarding the accuracy with which EELS spectra can be adequately calculated:

1) for each single NP, the probed chemical species lies in different atomic sites where they have different environments, the largest differences being between the bulk sites close to the centre of the object and the surface sites. The complete response of an NP will then be an average of all atoms responses, weighted by the number of equivalent sites in the object.

2) Generally speaking the environment of each atom in the NP is anisotropic: this is especially true for atoms close to the outer surface which have less neighbours than those near the centre. Calculation of these contributions must consequently account for such lowsymmetry atomic environments.

3) Unless epitaxial deposition is performed, the NPs may lie with more or less random crystallographic orientations on the supporting film. In principle, a second level of averaging is then required to calculate the statistical response of a whole population, unless it is demon- strated that individual responses are sufficiently similar, whatever the orientation of the particles.

Obviously some of the above considerations can be ignored if individual NPs are probed separately. This is however rarely possible:

(i) when the density of deposited NPs is high, or simply because of electrostatic or surface tension effects promoting the formation of agglomerates, overlap problems prevent an easy selection of 'isolated' objects. This is the common situation for NPs particles synthesized via a chemical route and deposited under the form of a drop of dilute suspension onto a carbon grid, as in the case of the $\mathrm{Gd}_{2} \mathrm{O}_{3}$ NPs studied in the present work.

(ii) In the most general case of a crystal with low symmetry, the EELS calculation requires the knowledge of the incident beam direction with respect to the crystal lattice (see section 2.4). This cannot be easily performed unless systematic nano-diffraction patterns are acquired, which remains very tedious for very small NPs. Moreover, high resolution lattice images may also permit the identification of zone axis as far as crosslattice fringes are effectively resolved, but this is again not easy in the case of small NPs.

(iii) For very small NPs, say below 3 to $4 \mathrm{~nm}$ in size, experimental difficulties frequently arise such as a low signal-to-noise ratio, mechanical instabilities or irradiation damage of the NP under the electron beam. The latter limitation is indeed very drastic if one aims 

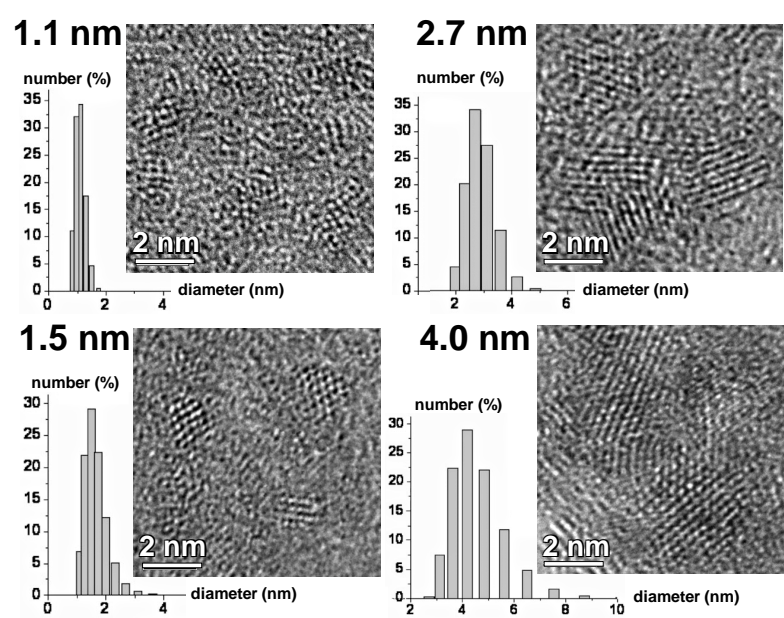

$4.0 \mathrm{~nm}$

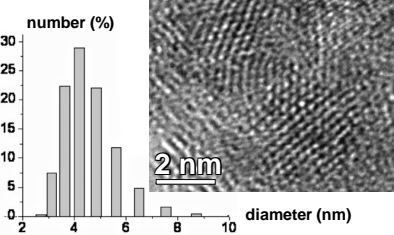

Fig. 1. HREM imaging of various calibrated samples of $\mathrm{Gd}_{2} \mathrm{O}_{3}$ NPs (see [8] for more details)

at using Angström or sub-Angström electron probes, which generally drill holes very rapidly.

\section{$1.2 \mathrm{Gd}_{2} \mathrm{O}_{3} \mathrm{NPs}$}

In the present paper, we have used $\mathrm{Gd}_{2} \mathrm{O}_{3}$ NPs, wellcalibrated in size, which were recently studied by EELS [8]. Gadolinium oxide NPs belong to this class of promising compounds to be used as magnetic contrast agents for medical imaging [9], or as inorganic cores of hybrid-type systems for medical applications (i.e. drug delivery and/or cancer therapy, see, for instance among recent overviews, [10-14]). Among these objects mixing organic and inorganic materials, gadolinium oxide particles are promising compounds. Typical High Resolution Electron Microscopy (HREM) micrographs obtained on a JEOL 2010F instrument, operated at $200 \mathrm{kV}$ and equipped with a Gatan DigiPEELS system, are presented in figure 1.
It was shown that the O-K edge varies with the size of the particles [8], and this feature will be examined using Electron energy-Loss Near-Edge Structure (ELNES) simulations accounting for steps 1) to 3) previously enumerated. These calculations were performed using the feff code based on a multiplescattering approach $[15,16]$. Section 2 details how this program can be used to simulate EELS spectra from a collection of randomly distributed particles, whereas the size effect will be illustrated in Section 3. Section 4 will then discuss and summarize the results.

\section{Feff calculations for $\mathrm{Gd}_{2} \mathrm{O}_{3}$ nano-particles}

Many computational codes allow one to calculate the double differential scattering cross section DDSCS. The subroutine TELNES of the Wien2k code using a band structure approach $[17,18]$ can be applied to true crystalline materials, or isolated objects artificially extended as super cells, which are then described by a periodic potential. Using periodic boundary conditions and the periodicity of the potential, the electron wave functions are described by Bloch waves. NPs are a totally different kind of materials for which this code cannot be used. Castep [19] and LMTO [20] codes also allows one to calculate ELNES spectra only for a periodic system of electrons and thus are not suited to the study of NPs. Finally, we have chosen the feff8.2 code $[15,16]$ which is better suited to such systems, as will be detailed in the following sub-sections. 


\subsection{Surface atoms and bulk atoms}

The study by ELNES of a material raises particular problems when this material is in the form of NPs. These problems come from the large range of non equivalent probed atom sites. Two main situations can thus be considered, according to the site location of the probed atom:

- The distance from the probed atom to the NP surface is large enough to make its geometrical environment and its local density of states similar to those of a bulk atom. This situation can be encountered for central atoms in spherical particles with a rather large radius.

- The probed atom site is too close to the surface to be considered as a bulk atom. In the case of very small NPs, most of the probed atoms cannot be considered as bulk atoms, so, the DDSCS associated with a particular probed atom depends on its location inside the NP. Actually, for the case of $\mathrm{O}$ in $\mathrm{Gd}_{2} \mathrm{O}_{3}$ NPs considered in the present work, the DDSCS should be calculated for all the possible probed $\mathrm{O}$ sites, and the average over these results should be considered. Because the number of nonequivalent $\mathrm{O}$ sites is very large, we might be led to calculate a very large number of DDSCS. The volume of this calculation can be markedly reduced by making a rough distinction between bulk and surface atoms.

\subsection{Use of the feff8. 2 code}

In a classical feff calculation, the probed material is described by an atomic cluster and the probed atom $(\mathrm{O}$ in the present investigation) is located at the centre of the cluster which is chosen as the origin of space. Three atomic scattering potentials, characterized by a number ipot in the feff code[15], will then be self-consistently calculated in the $\mathrm{Gd}_{2} \mathrm{O}_{3}$ cluster:

- the potential of the probed oxygen atom $($ ipot $=0)$;

- the potential of a gadolinium atom located near the probed atom $($ ipot $=1)$;

- the potential of an oxygen atom located near the probed atom $($ ipot $=2)$.

So, taking the partial $1 s$ core level occupancy of the probed oxygen into account, the scattering potential of the probed atom has to be treated separately. Although the atomic environment of bulk and surface atoms can be very different, their scattering potentials are usually assumed to be identical in feff calculations. This assumption seems to be unsuitable, particularly when the probed atom site is located near the NP surface. This difficulty can be partially overcome by making a distinction between surface atoms and bulk atoms. In the case of the body-centred cubic lattice of the $\mathrm{Gd}_{2} \mathrm{O}_{3}$ oxide (space group Ia3, with $\mathrm{a}=1.08 \mathrm{~nm})[21]$, a bulk oxygen atom has four nearest neighbour Gd atoms. On the other hand, in an NP, this number can vary from four to one. In order to discriminate bulk and surface $\mathrm{O}$ atoms, we adopt the following convention: an $\mathrm{O}$ atom is assumed to be a surface atom when it is surrounded with one or two Gd atoms, while it is considered as a bulk atom when it is surrounded with three or four Gd atoms. These two kinds of oxygen atoms are treated as full species. In the same way, a bulk Gd has 
six nearest neighbour $\mathrm{O}$ atoms, whereas, in an NP, this number can vary from six to two. We adopt the following convention: a Gd atom is assumed to be a surface atom when it is surrounded with three or less $\mathrm{O}$ atoms, while it is a bulk atom when it is surrounded with four or more $\mathrm{O}$ atoms. As in the case of the oxygen atoms, both kinds of Gd atoms are treated as if they were different species ${ }^{1}$.

The atoms program of Bruce Ravel [15] generates an input file, feff.inp, in which the probed $\mathrm{O}$ atom is at the centre of an atomic cluster, which defines the origin of space for the feff calculation. Since we have to treat the ionization of any atom at any $\mathrm{O}$ site within the NP, and not only at the centre of the NP, the feff.inp file obtained from the atoms code is modified to set the origin of space on the chosen $\mathrm{O}$ site, occupied by the probed ionized atom with ipot $=0$. The list of atoms in the ATOMS card is then sorted according to their increasing distances from the new probed $\mathrm{O}$ site. Their ipot value is assigned to $i$ (1 to 4), depending upon their 'nature' as described above. A geometrical illustration of this description is depicted in figure 2. This figure shows projections of a typical $\mathrm{Gd}_{2} \mathrm{O}_{3}$ cluster with a radius of $4.91 \AA$ and containing 37 atoms, corresponding to the true composition $\mathrm{Gd}_{2} \mathrm{O}_{2.9}$. $\mathrm{O}$ and Gd atoms are represented with large red and small blue circles respectively. The configuration on the left shows

\footnotetext{
${ }^{1}$ the ATOMS card of the input file, feff.inp, contains a flag ipot which can take five values: always 0 for the probed atom (here, a $\mathrm{O}$ atom) and from 1 to 4 for both kinds of $\mathrm{O}$ and $\mathrm{Gd}$ atoms.
}

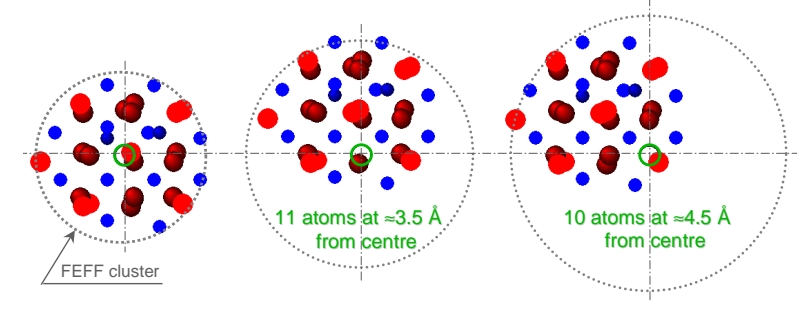

Fig. 2. $\mathrm{Gd}_{2} \mathrm{O}_{3}$ clusters with a radius of $4.91 \AA$ and containing 37 atoms.

the probed $\mathrm{O}$ site (the green circle) at the centre of the NP. In the central figure, the probed atom is one of the 11 $\mathrm{O}$ atoms located at about $3.5 \AA$ from the NP centre. The right figure shows a situation where the probed $\mathrm{O}$ is one of the $10 \mathrm{O}$ located at about $4.5 \AA$ from the NP centre. Note that these surface $\mathrm{O}$ atoms represent about $45 \%$ of the total number of $\mathrm{O}$ atoms in the NP. In all displays, shaded atoms are considered as bulk $\mathrm{O}$ atoms (i.e. with three or more than three nearest neighbours) whereas filled ones are surface $\mathrm{O}$ atoms (with two or less than two nearest neighbours). The outer dotted circle represents the calculated cluster in feff.

\subsection{The anisotropy of the differential cross section}

The DDSCS associated with a particular bulk O site is rather complicated because its symmetry is very low. A bulk O site looks like a tetrahedral site. However, the four Gd sites around the bulk $\mathrm{O}$ site do not form a regular tetrahedron: they are actually located at four different distances from the $\mathrm{O}$ site. Let us also point out that there are two kinds of octahedral Gd bulk site. However, one of them is not associated with a regular octahedron. The symmetry of a bulk $\mathrm{O}$ site (and a fortiori of a surface 
O site), is such that neither a many-fold rotation axis, nor a plane of symmetry passes through this site. As a consequence, we are in a situation of $c$ trichroism [22]: the DDSCS, for a particular orientation of the transferred wave vector $\mathbf{q}$, can be expressed as a linear combination of six DDSCSs called intrinsic components [22], calculated for the [001], [010], [011], [100], [101] and [110] orientations of the incident and transferred wave vectors, $\mathbf{k}$ and $\mathbf{q}=-\theta_{E} \mathbf{k}$ respectively $\left(\theta_{E}\right.$ is the characteristic angle). It is important to understand that an intrinsic component is a cross-section corresponding to a transfer momentum $\mathbf{q}$ strictly parallel to the incident beam direction $\mathbf{k}$ along a particular direction.

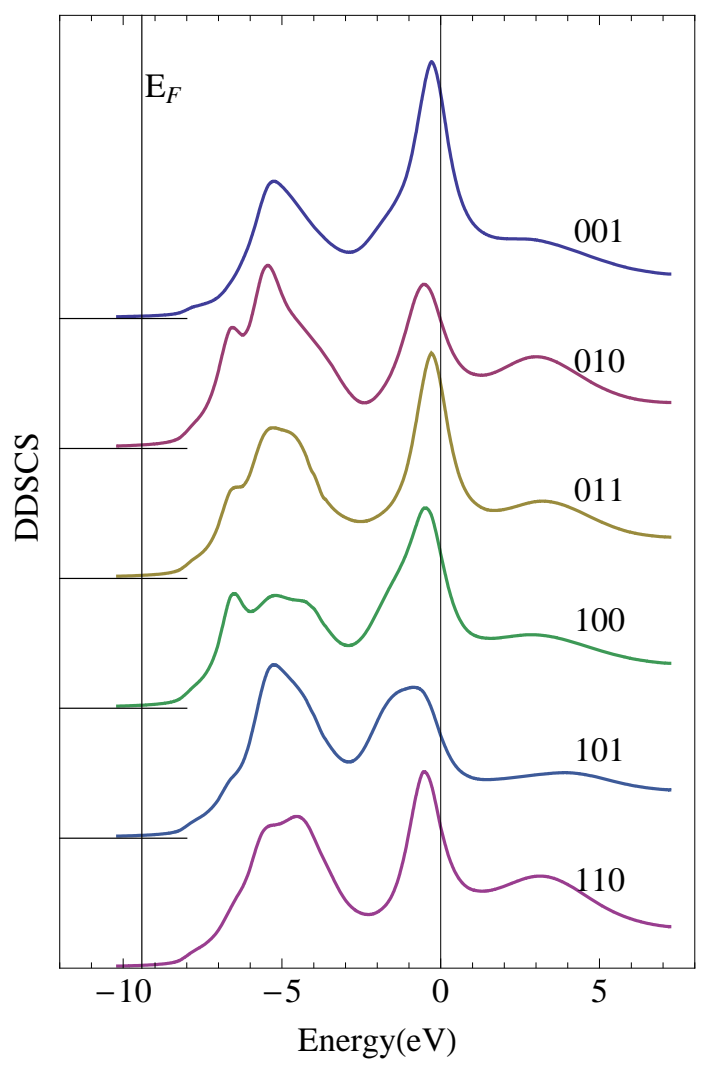

Fig. 3. Intrinsic components for the feff cluster shown in figure 2
Using of the POLARIZATION card in the feff code allows calculating the DDSCS for these six particular orientations of q. For instance, figure 3 shows these intrinsic components in the case where the probed $\mathrm{O}$ site is located at the centre of an atomic cluster of 37 atoms. The bulk $\mathrm{O}$ site is very close to a tetrahedral site, for which the DDSCS would be isotropic (under the dipole approximation), which means that the six intrinsic components would be equal. Figure 3 clearly shows that a slight distortion of the regular tetrahedron made up of the four Gd atoms, induces a marked dependence of the DDSCS on the orientation of the transferred wave vector. Particularly, the DDSCS for q parallel to the [001] and [101] directions are very different.

Let us notice that this plot is carried out by choosing the origin of energy at the vacuum energy. Actually, in all further developments, ELNES or DDSCS will be plotted by choosing the vacuum energy rather than the $1 s$ core level of the $\mathrm{O}$ atom as the origin of energy. The 1s core level of an $\mathrm{O}$ atom indeed depends on its atomic neighbourhood. Particularly, it is deeper the larger the number of its nearest Gd neighbours. Therefore, the $1 s$ core level of an $\mathrm{O}$ atom depends on its location inside the cluster and cannot be considered for a reference of energy.

\subsection{ELNES signal for a probed oxygen site}

It has been shown that the ELNES signal obtained with a particular direction of the incident beam, is also a weighted average of the six intrinsic components previously defined [22]. 
However, the weights of these components are different from those of the DDSCS. They depend on the incident beam orientation, but also on the collection aperture, and the convergence angle of the incident beam [22]. We are in an experimental situation where the collection aperture $\left(\beta_{m}=7 \mathrm{mrad}\right)$ and the convergence angle of the incident beam $\left(\alpha_{m}=10 \mathrm{mrad}\right)$ are large compared with the characteristic angle $\left(\theta_{E} \approx 1.57 \mathrm{mrad}\right)$. These weights were calculated for different orientations of the incident beam, and the results are reported in table 1 . It can be observed that the three weights $k_{001}, k_{010}$ and $k_{001}$ have generally a dominant contribution.

For instance, let us now consider a cluster with 86 atoms which contains the probed oxygen at its centre. This cluster is very similar to the NP experimentally studied in a previous paper [8]. Figure 4 shows the six intrinsic components used in the case of $c$ trichroism (see section 2.3). Let us point out that these six intrinsic components are noticeably different from those of figure 2, obtained for an $\mathrm{O}$ atom at the centre of a cluster of 37 atoms (this point will be briefly re-discussed below).

The simulated ELNES signals of a probed $\mathrm{O}$ atom are plotted in figure 5 for two different locations of its site and for different orientations of the incident beam. These simulations require calculation of the weights of intrinsic components for these different orientations (their expression can be found in ref. [22]). According to the experimental acquisition conditions, they were evaluated for a
Table 1. Weights of intrinsic components for different orientations of a $200 \mathrm{keV}$ incident beam. Using the spherical coordinates, these orientations are defined by the colatitude angle, $\chi_{0}$, and the longitude angle, $\delta_{0}$, according to the choice of axes in the atoms program [15].

\begin{tabular}{|c|c|c|c|c|c|c|}
\hline & $k_{100}$ & $k_{010}$ & $k_{001}$ & $k_{110}$ & $k_{011}$ & $k_{101}$ \\
\hline $\begin{array}{l}\chi_{0}=0 \\
\delta_{0}=0\end{array}$ & 0.43 & 0.43 & 0.14 & 0.0 & 0.0 & 0.0 \\
\hline $\begin{array}{c}\chi_{0}=45 \\
\delta_{0}=0\end{array}$ & 0.43 & 0.43 & 0.43 & 0.0 & 0.0 & -0.29 \\
\hline $\begin{array}{c}\chi_{0}=90 \\
\delta_{0}=0\end{array}$ & 0.14 & 0.43 & 0.43 & 0.0 & 0.0 & 0.0 \\
\hline $\begin{array}{c}\chi_{0}=30 \\
\delta_{0}=45\end{array}$ & 0.52 & 0.52 & 0.39 & -0.07 & -0.18 & -0.18 \\
\hline $\begin{array}{c}\chi_{0}=45 \\
\delta_{0}=90\end{array}$ & 0.43 & 0.43 & 0.43 & 0.0 & -0.29 & 0.0 \\
\hline
\end{tabular}

collection angle $\beta_{m}=7 \mathrm{mrad}$, and an incident beam convergence angle $\alpha_{m}=10 \mathrm{mrad}$. For reasons explained below, the arithmetic average (denoted by average) of the three intrinsic components, [001], [010] and [100] is also reported in figure 5. A quick examination of simulated spectra allows us to conclude that their shape:

1) is not very sensitive to the incident beam orientation; 

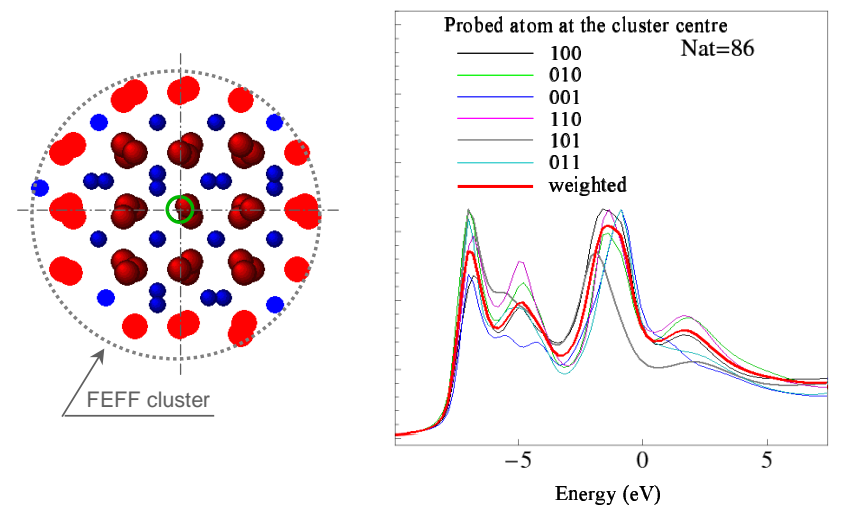

Fig. 4. On the left, this figure shows a cluster of 86 atoms with a radius equal to $7.05 \AA$ and corresponding to the almost stoichiometric composition, $\mathrm{Gd}_{34} \mathrm{O}_{52}$. The probed $\mathrm{O}$ is at the centre of this cluster. On the right, the six superimposed intrinsic components corresponding to the situation of $c$ trichroism are shown. As the DDSCS, the ELNES signal (denoted by weighted) calculated when the incident beam is parallel to the [001] direction is a weighted average of these intrinsic components. For the ELNES signal recorded in this orientation, the six weights $k_{m n p}$ are given at the first line of table 1 ; they are markedly different from those corresponding to the DDSCS obtained in the [001] direction, that is: $k_{001}^{0}=1$, and all other weights equal to zero.

2 ) is very sensitive to the location of the probed $\mathrm{O}$ site in the cluster.

It is easy to understand that if the six intrinsic components are not markedly different (see figure 4), then the ELNES signal (i. e. a weighted average of these components) can show a marked dependence on the incident beam orientation, only if the weights strongly vary with this orientation. Table 1 indicates that, for the acquisition conditions used here, the weights of intrinsic components do not show marked changes with the beam orientation, thus
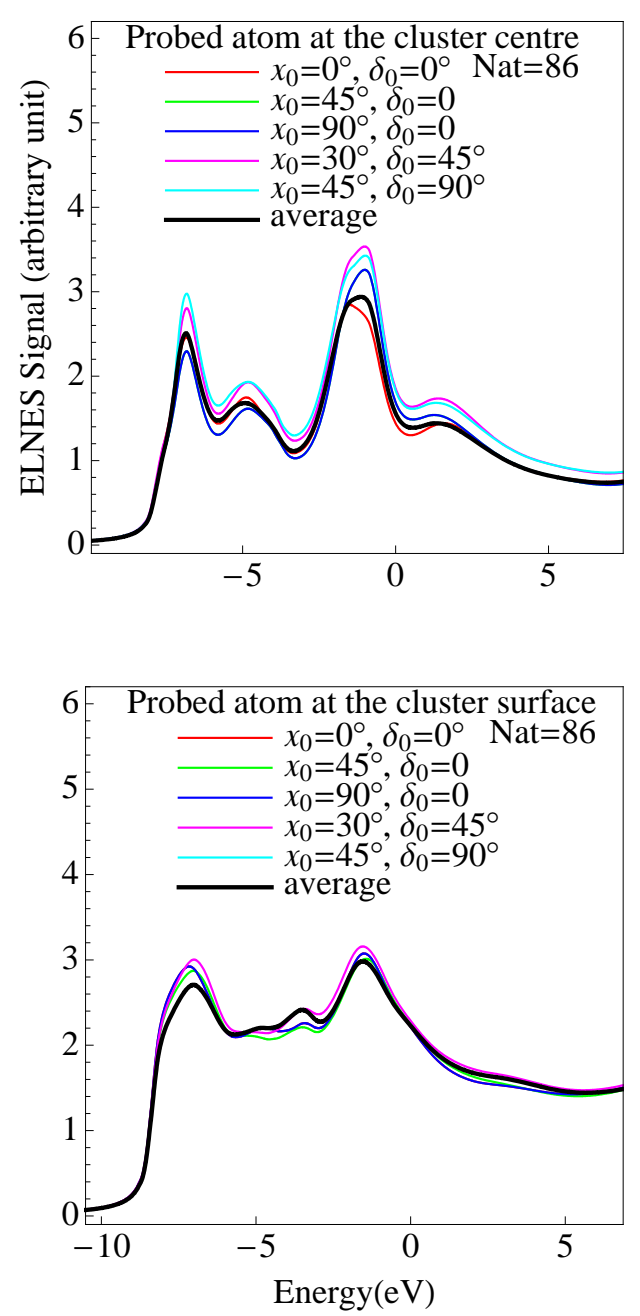

Fig. 5. Simulated ELNES signal obtained for a NP of 86 atoms for different orientations of the incident beam. Top figure: the probed $\mathrm{O}$ site is at the cluster centre, bottom figure: the probed O site is at $4 \AA$ from the NP centre. These spectra are not very incident beam dependent, and can be replaced with the average obtained with the weights $(1 / 3,1 / 3,1 / 3,0,0,0)$.

explaining the first conclusion (1) above. This also proves that, at least in the present case, the resulting ELNES from a collection of particles with the same size will not depend on their relative orientation: this means that any texture, or preferential orientation of the NPs on the supporting film (which is actually not the case here, owing 
to the nature and preparation method of the TEM sample) would not influence the results. Conversely, the first conclusion would not be true if the incident beam convergence, $\alpha_{m}$, and the collection aperture, $\beta_{m}$, were similar to or less than the characteristic angle $\left(\theta_{E} \approx 1.57 \mathrm{mrad}\right)$. The DDSCS and simulated ELNES spectra are a linear combination of the six intrinsic components [22], but the weights for the DDSCS are very different from those of the ELNES signal. For example, let us consider the two cases where the beam direction is $[001]\left(\chi_{0}=0\right)$ or $[101]$ $\left(\chi_{0}=45^{\circ}\right.$ and $\left.\delta_{0}=0\right)$. The sum of weights being always 1 , if the incident beam orientation goes from the [001] to the [101] direction, then the DDSCS weight $k_{001}^{0}$ varies from 1 to 0 , and $k_{101}^{0}$ varies from 0 to 1 , while the ELNES signal weight $k_{001}$ varies from 0.14 to 0.43 , and surprisingly ${ }^{2} k_{101}$ varies from 0.0 to -0.29 (see table 1 ). It can be concluded that the dependence on the beam orientation of the DDSCS weights is larger than that of the simulated ELNES signal. Accordingly, the examination of figs. 3 and 5, clearly shows that the anisotropy of the simulated ELNES signal is small compared with that of the

\footnotetext{
${ }^{2}$ If $\alpha_{m}$ and $\beta_{m}$ approaches $\theta_{E}$, then $k_{001}=k_{010}=k_{001}$ decreases from 0.43 to 0.29 , while $k_{101}$ increases from -0.29 to 0.13 . If $\alpha_{m}$ and $\beta_{m}$ are about $\theta_{E} / 2$, then $k_{001}=k_{010}=$ $k_{001} \approx 0.18$ and $k_{101} \approx 0.46$. To get the DDSCS weights, that is to say to be able to measure the [101] intrinsic component it would be required to deal with a parallel incident beam and a very small collection aperture, which leads to an undetectable ELNES signal.
}

DDSCS.

Moreover, the DDSCS obtained for a transferred wave vector $\mathbf{q}=-\theta_{E} \mathbf{k}$ parallel to the incident beam direction is a poor approximation of a simulated ELNES spectrum. In this case, it is noteworthy to see that, though the incident beam is parallel to the [001] direction, $k_{001}=0.14$ is smaller than all other non zero components: $k_{010}=$ $k_{100}=0.43$. Generally, the ELNES signal cannot be obtained from a DDSCS calculation in which the transferred wave vector $\mathbf{q}=-\theta_{E} \mathbf{k}$ is parallel to the incident beam direction.

It can be easily understood that the closer the probed atom site is to the NP surface, the larger the anisotropy of the intrinsic components. However, figure 5 illustrates a general trend observed here: the simulated ELNES signal remains almost independent of the incident beam direction, even if the probed $\mathrm{O}$ atom site is a surface site, i.e. the intrinsic components are strongly anisotropic. In all cases examined here, it can be observed that the arithmetic average of [001], [010] and [100] components remains indeed very close to the simulated ELNES signal from the NP calculated for different beam orientations. This is for example clearly evidenced by comparing this average in the case of the central atom from the 86 atoms $\mathrm{NP}$ of fig. 5 to the exact linear combination depicted in Figure 4. This conclusion is of importance, because the experimental determination of the exact crystal orientation is very difficult. Thanks to this result, this difficulty can be overcome by replacing the simulated ELNES spectrum with 
this arithmetic average.

\subsection{ELNES signal for a nano-particle}

The calculation of ELNES spectra for all the possible probed $\mathrm{O}$ sites of the cluster representing a NP is a very heavy calculation. We can describe the cluster as a stacking of $j$ concentric $\mathrm{O}$ shells, each at a distance $d_{j}$ from the $\mathrm{NP}$ centre (that is, an atomic shell contains $\mathrm{O}$ sites located at a distance between $d_{j}-0.5$ and $d_{j}+0.5 \AA$ from the centre). Instead of calculating the ELNES contribution from each atom within each shell, we restrict ourselves to a representative sampling of these $\mathrm{O}$ sites: a probed atom in any $d_{j}$ shell is chosen in such a way it is the most representative O site of this shell, i. e. its atomic neighbourhood has to be the most frequently encountered. Then, the total ELNES signal for the NP is assumed to be an average of these representative ELNES spectra, weighted by the number of $\mathrm{O}$ atoms in each shell. For instance, the 86 atoms cluster shown in Figure 4 can be separated into five shells: the first shell contains $1 \mathrm{O}$ site at less than $3 \AA$ from the centre, the second shell contains $11 \mathrm{O}$ sites between 3 and $4 \AA$ from the centre, the third shell contains $10 \mathrm{O}$ sites between 4 and $5 \AA$, the fourth shell contains $10 \mathrm{O}$ sites between 5 and $6 \AA$ and the fifth shell contains $20 \mathrm{O}$ sites between 6 and $7.05 \AA$. One O probed site has to be chosen in each shell, and five ELNES spectra corresponding to these five probed $\mathrm{O}$ sites have to be calculated. Then, the simulated ELNES signal for this NP is assumed to be a weighted average of these five ELNES spectra. In the

\begin{tabular}{||c|c|c|c|c||}
\hline \hline & size & $N_{a t}$ & $N_{O}$ & $N_{G d}$ \\
\hline cluster 1 & $4.91 \AA$ & 37 & 22 & 15 \\
\hline cluster 2 & $7.05 \AA$ & 86 & 52 & 34 \\
\hline cluster 3 & $7.80 \AA$ & 120 & 72 & 48 \\
\hline cluster 4 & $10.11 \AA$ & 405 & 243 & 162 \\
\hline \hline
\end{tabular}

Table 2. Definition of the atomic cluster used in ELNES simulations.

present case, the weights are $1 / 52,11 / 52,10 / 52,10 / 52$ and $20 / 52$.

\section{Size effects}

The size effects are investigated by repeating this kind of calculation for several atomic clusters with different diameters. The choice of these clusters is done so that the ratio of the number of $\mathrm{Gd}$ to $\mathrm{O}$ atoms is as close as possible to $2 / 3$. The size of an atomic cluster is defined as the distance from its central atomic site to the most distant atomic site. Here, we have investigated four atomic clusters of which the size and atom numbers are given in table 2 . These simulations are compared with two measured ELNES spectra obtained from two sets of NPs. These spectra are the results of the averaging of several measurements obtained on different areas, each including several particles. Although the size of each individual NP cannot be measured accurately, data reported in figure 1 indicate they have a homogeneous and narrow size distribution with reasonably spherical shapes. A selection of NPs with respective diameters of some $1 \mathrm{~nm}$ (NPs referenced as a) and 1.5 
nm (NPs referenced as b) has thus been done. The simulations of ELNES spectra (thin lines) are represented in figure 6 together with the experimental results (dashed lines). These simulations are broadened by a convolution
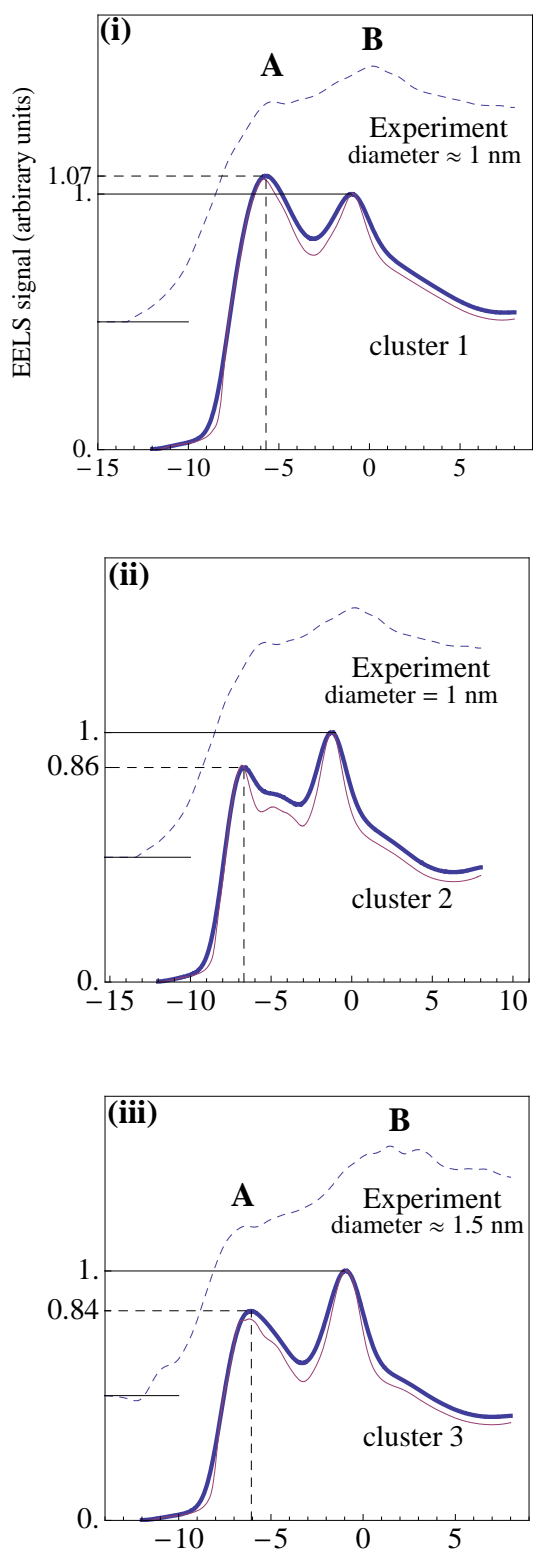

with a Gaussian of $1.2 \mathrm{eV}$ FWHM (thick line).

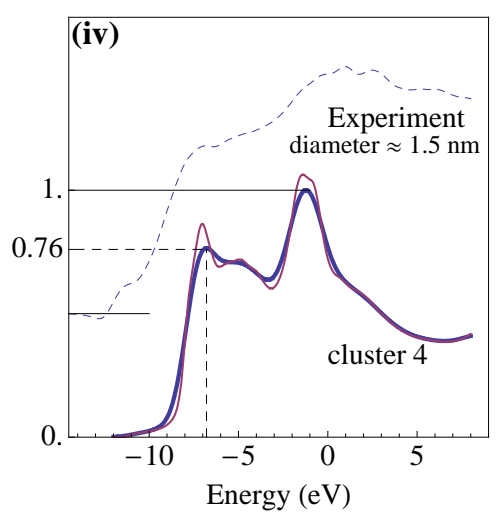

Fig. 6. Figures (i), (ii), (iii) and (iv) show the ELNES signal measured in the case of two populations of NPs $a$ and $b$ (dashed line). Simulations of the ELNES signal for four atomic clusters are reported (thin line). The size and the number of atoms in these clusters are given in table 2

The measured ELNES spectra display two structures: a pre-peak $A$ located at about $-6 \mathrm{eV}$ and a peak $B$ located at about $0 \mathrm{eV}$ for the NPs $a$ and $2 \mathrm{eV}$ for the NPs $b$. It can be observed that the height of the pre-peak A decreases when the NP diameter increases, whereas the separation between pre-peak $A$ and peak $B$ increases. It can also be observed that the simulations for the four clusters also present a pre-peak and a peak. The same trends as those observed in the experiment are obtained: the height of the pre-peak decreases and the separation between both peaks increases when the cluster size increases.

Obviously one may wonder about the possible influence of the solvent and its undesirable contribution to the O-K edge of interest. Initially the particles are colloidally stable in a solution of $20 \mathrm{mmol}$ of gadolinium per litre of diethylene glycol (DEG). When drops of solution were deposited on the TEM grids, it is highly likely that 
some spurious solvent remains around the particles, although the major part was eliminated by the use of an absorbing tissue, and further evaporated in the pumping system of the microscope. Figure 7 demonstrates however that this spurious contamination is clearly negligible. A typical spectrum from a set of NPs is compared to that from the carbon supporting film where the largest oxygen signal was detected, probably due to some remaining DEG layer. Both spectra were roughly normalized according to the carbon peak. It is seen that this oxygen contribution remains considerably lower than that from the NPs. Indeed further linear combinations of theses O-K edges, once the background subtracted, indicate that the fine structure variations attributed to the NPs size effects are more than ten times larger than this maximum spurious signal. This point certainly merits a deeper quantitative investigation of the coverage of NPs by a remaining DEG layer in terms of wetting effects (for example using a sub-Angström probe and very fast EELS acquisitions in a dedicated microscope - a delicate experiment as briefly discussed in the introduction section). Nevertheless the fact of greatest importance in the context of the present study is that we can neglect any spurious oxygen contribution when analyzing the size effect on the O-K ELNES features.

It can be seen that these modifications of the ELNES spectra are correlated with the change in the $2 p$ local density of states (LDOS) of the probed oxygen, when its site is displaced from the centre to the surface of the cluster (see figure 8 ). For both sites, the $2 p *$ LDOS presents a

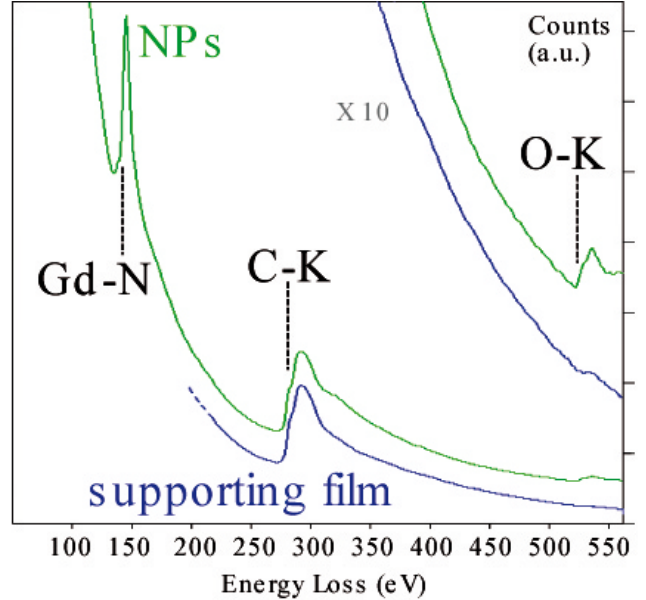

Fig. 7. Typical EELS spectra from a set of NPs (labelled NPs) and from the carbon supporting film, where some oxygen contamination is detected.

sharp peak around $-15 \mathrm{eV}$. This peak is shifted up when the probed site is displaced from the centre to the surface of the cluster. However, this displacement has no direct influence on the unoccupied LDOS located just above the Fermi level $\left(E_{F} \approx-8.0 \mathrm{eV}\right)$. For both sites, the occupation of the $2 p$ LDOS indicates a strong transfer of electrons from the $\mathrm{Gd}$ atoms to the $\mathrm{O}$ atoms. This electronic transfer was invoked to interpret the experimental EELS spectra previously reported[8]. In the case of both kinds of probed atom site, the LDOS just above the Fermi level and the DDSCS are very similar.

Finally, the decrease in the first pre-peak correlated with the LDOS change just above the Fermi level is the evidence of a size effect. In the case where the probed atom is located at a bulk site, the DDSCS displays two peaks, $A$ and $B$, but the peak $B$ is higher and wider than the prepeak $A$ (see figure 5 ). On the other hand, the DDSCS of a probed atom located near the surface displays two peaks 
$A$ and $B$ with a comparable height, but the pre-peak $A$ is wider than the peak $B$ (see figure 5 ). For a rather small cluster, where the number of surface $\mathrm{O}$ atoms is large compared with the number of bulk $\mathrm{O}$ atoms, the pre-peak $A$ is more prominent than in the case of a rather big cluster, where the number of $\mathrm{O}$ surface atoms is smaller than the number of $\mathrm{O}$ bulk atoms.

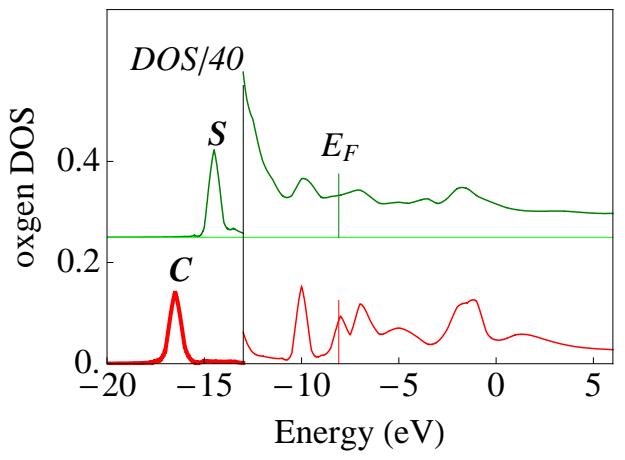

Fig. 8. This figure shows the $2 p$ local DOS (LDOS) of the probed atom when is site is at the centre of the cluster $(\mathrm{C})$ and at the surface of the cluster $(\mathrm{S})$. Under $-13 \mathrm{eV}$ the $2 p$ DOS presents a very sharp peak which can be represented only if its amplitude is divided by a factor 40 .

\section{Discussion and conclusion}

The present ELNES simulations have demonstrated that the EELS spectra are sensitive to the size of the NPs in the case of the $\mathrm{Gd}_{2} \mathrm{O}_{3}$ system. Firstly the DDSCS that is calculated for a particular probed atom depends on its location inside an NP, consequently the EELS signal is also site-dependent as was shown in figure 5. Secondly, DDSCSs for similar sites also vary with the cluster size, as can be seen by comparing calculations for central and surface atoms in the case of an NP of 86 atoms (figure 5) with similar calculations in the case of an NP of 37 atoms, as reported in figure 9. The comparison of both figures shows however that the predominant difference is related to the site position. As a consequence, in the range of the NP sizes considered here, the size effect observed in the ELNES spectrum of a whole NP depends mostly on the ratio of surface to bulk $\mathrm{O}$ atoms, rather than on the NP size, although both quantities are obviously related. This relation can be more complex than what was accounting in the present work, and further experimental and theoretical studies would certainly be profitable on model systems.

We can further emphasize that when the particle size is large enough to be able to assume that most of the possible probed atoms are located at geometrically equivalent sites, no size effect is observable. When this condition is not fulfilled, the number of atomic sites which can no longer be considered as bulk sites increases. There is a single scattering cause of the change in the DDSCS: the scattering potentials of species located at these sites are different from the scattering potential of the same species located at a bulk site. We have roughly taken this cause into account by considering bulk and surface atoms in a simplified, but realistic way (from the point of view of the simulations complexity). As the atomic environments of surface and bulk atoms are different, the DDSCSs of surface and bulk probed atoms are different too, but this difference is a consequence of multiple scattering processes. This dominant cause is underscored by comparing the DDSCSs obtained 

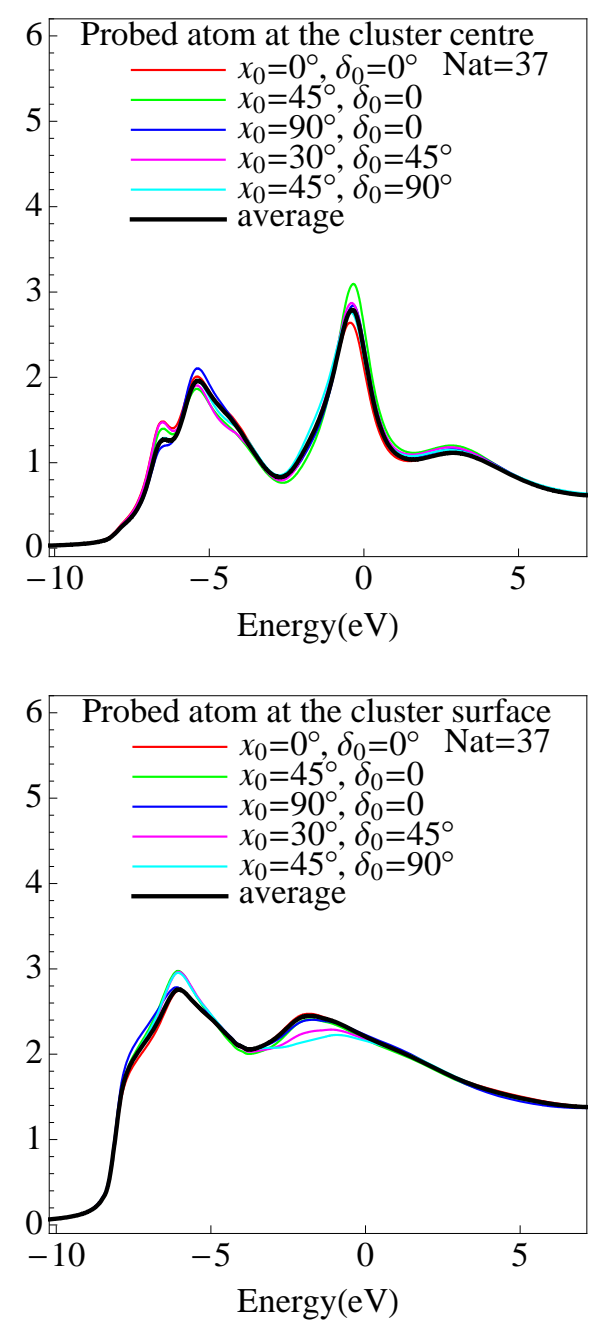

Fig. 9. Simulated ELNES signal obtained for a NP of 37 atoms for different orientations of the incident beam. Top figure: the probed $\mathrm{O}$ site is at the cluster centre, bottom figure: the probed $\mathrm{O}$ site is at $4 \AA$ from the NP centre. As for the case of the cluster of 86 atoms reported in figure 5 , these spectra are not very incident beam dependent, and can be replaced with the average obtained with the weights $(1 / 3,1 / 3,1 / 3,0,0,0)$.

by a feff calculation for a probed $\mathrm{O}$ atom located at a bulk site and another one at a surface site. One takes into account this effect due to multiple scattering by taking the average of the DDSCSs calculated for a set of the different probed atom sites encountered in the sample. Considering single and multiple scattering effects leads to results in a fair agreement with experiment. Let us notice that these results would be probably in a better agreement with experiment if we could take into account the actual surface barrier potential of the NP.

\section{Acknowledgements}

The FEFF8.2 calculations were performed at the Centre Informatique National de l'Enseignement Supérieur (CINES): gep2532 project. Thanks are due to the CLYM (Centre Lyonnais de Microscopie) for the access to the TEM. Vincent Mauchamp (PhyMat laboratory, CNRS UMR 6630, Poitiers University, F) is gratefully acknowledged for his contribution to the EELS experimental work.

\section{References}

1. P. Buffat, J. P. Borel, Physical Review A, 13, 6, (1976), $2287-2298$.

2. W. H. Qi, M. P. Wang, J. of Mat. Sci. Letters, 21, (2002), 1743-1745.

3. D. Alloyeau, C. Ricolleau, C. Mottet, T. Oikawa, C. Langlois, C. Langlois, Y. Le Bouar, N. Braidy, A. Loiseau, Nature Materials, 8, 12, (2009), 940-946.

4. G. A. Somorjai and J. Y. Park, Topics in Catalysis, 49, 3-4, (2008), 126-135.

5. J. Jiang, G. Oberdrster and P. Biswas, J. Nanopart. Res., 11, (2009), 7789 . 
6. J. Nelayah, M. Kociak, O. Stephan, N. Geuquet, L. Henrard, F. J. Garciá de Abajo, I. Pastoriza-Santos, L. M. LizMarzań and C. Colliex, Nano letters, 10, 3, (2010), 902-907.

7. B. Schaffer, W. Grogger, G. Kothleitner and F. Hofer, Ultramicroscopy, 110, 8, (2010), 1087-1093.

8. M. Ou, V. Mauchamp, B. Mutelet, T Épicier, J. C. Le Bossé, S. Roux, O. Tillement and P. Perriat, J. Phys. Chem. C113(10), (2009), 4038-4041.

9. J. L. Bridot, A. Faure, S. Laurent, C. Riviere, C. Billotey, B. Hiba, M. Janier, V. Josserand, J. L. Coll, L. Vander Elst, R. Muller, S. Roux, P. Perriat, O. Tillement, J. Am. Chem. Soc. 129, (2007), 5076-5084.

10. F. X. Gu, R. Karnik, A. Z. Wang, F. Alexis, E. LevyNissenbaum, S. Hong, R. S. Langer and O. C. Farokhzad, Nanotoday, 2, 3, (2007), 14-21.

11. C. M. J. Hu, L. Zhang, Current Drug Metabolism, 10, 8, (2009), 836-841.

12. K. Subramani, H. Hosseinkhani, A. Khraisat, M. Hosseinkhani, Y. Pathak, Current Nanoscience, 5, 2, (2009), 135140.

13. C. Tang, P. J. Russell, R. Martiniello-Wilks, J. E. J. Rasko, A. Khatri, STEM CELLS, 28, 9, (2010), 1686-1702.

14. S. Jiang, M. K. Gnanasammandhan, K. Muthu, Y. Zhang, J. of the Roy. Soc. Interface, 7, 42, (2010), 3-18.

15. A. L. Ankudinov, B. Ravel, J. J. Rehr and S. D. Conradson, Phys. Rev. B 58 (1998), 7565.

16. A. L. Ankudinov, C. E. Bouldin, J. J. Rehr, J. Sims and H. Hung, Phys. Rev. B 65 (2002), 104107

17. P. Blaha, K. Schwarz, G. Madsen, D. Kvanischka and J. Luiz, WIEN2k, An Augmented Plane Wave Plus Local Orbitals Program for Calculationg Crystal Properties, edited by K. Schwarz, Technical Universität Wien, Austria, 2001.
18. C. Hébert, Micron, 38, 1, (2007), 12-28.

19. S. Ostanin, A.J. Craven, D.W. McComb, D. Vlachos, A. Alavi, M.W. Finnis and A.T. Paxton Phys.Rev. B bf 62, (2000), 14728.

20. R. Tank, O. Jepsen, A. Burkhardt, and O. K. Andersen, The TB-LMTO- ASA Programm 1995, LMTO 47 manual.

21. V. Grover, S.N. Achary and A.K. Tyagi, J. Appl. Cryst. 36 (2003), 1082-1084

22. J. C. Le Bossé, T. Épicier and H. Chermette, Phys. Rev. B 76, (2007), 075127. 